(C) N. Babudri ${ }^{1}$, A. Lucaccioni ${ }^{1}$, A. Achilli ${ }^{2}$

${ }^{1}$ Dipartimento di Biologia Cellulare e Ambientale,

Perugia, Italy

${ }^{2}$ Dipartimento di Genetica e

Microbiologia,

Pavia, Italy

The nature of mutation in microorganisms has been debated for a long time. Two theories have been at odds: random spontaneous mutagenesis vs. adaptive mutagenesis. "Random mutagenesis" means that mutations occur in proliferating cells before they encountered the selective agent. "Adaptive mutagenesis" means that advantageous mutations form in the environment where they have been selected, in non-replicating or poorly replicating cells even though other, non-selected, mutations occur at the same time. In the last 20 years it has been definitely shown that random as well as adaptive mutagenesis occur in bacteria and yeast. Microorganisms in nature do not divide or divide poorly because of adverse environmental conditions; therefore adaptive mutations could provide cells with a selective advantage and allow evolution of populations. Here we will focus on some fundamental aspects of adaptive mutagenesis in the yeast Saccharomyces cerevisiae. We begin with a historical overview on the nature of mutation. We then focus on experimental systems aimed at proving or disproving adaptive mutagenesis. We have briefly summarized the results obtained in this field, with particular attention to genetic and molecular mechanisms.

Key words: adaptive mutagenesis, directed mutagenesis, DNA repair, yeast, resting cells, Saccharomyces cerevisiae

\section{ADAPTIVE MUTAGENESIS IN THE YEAST SACCHAROMYCES CEREVISIAE}

\section{INTRODUCTION}

For many years bacteriologists debated the idea that bacteria can adapt to new environmental conditions and that the acquired characters may become hereditary. Indeed some experimental data suggested that a Lamarckian evolution could take place in bacterial populations. An example has been reported by Hayes [1] on the coliform bacillus Escherichia coli mutabile. Cells of this microorganism are unable to ferment lactose (Lac-) and retain this inability indefinitely when cultivated without this sugar. However, when they are transferred to a lactosecontaining medium, fermentation begins after about one day; if at this point, these cells are sub-cultured in a lactose medium, fermentation starts at once. This ability to ferment lactose without delay is stably retained. In bacteria this behavior is also typical in the case of nutritional characters and resistance to antibiotics. On the basis of these observations many bacteriologists suggested that bacterial variation does not result from random events but from adaptation to the environment. In 1943 Luria and Delbrück [2] tested this hypothesis by the fluctuation test: they analyzed the distribution of bacteriophage T1 resistant colonies in independent cultures of $\mathrm{T} 1$ sensitive bacteria by plating on selective medium. A series of 5 to 100 cultures were set up in parallel with small inocula. This was to make sure that resistant bacteria were not introduced into the cultures at the beginning of the experiment. The cultures were grown until maximum titer was reached. Then they were plated on the selective plates containing the bacteriophage: on this medium only $\mathrm{T} 1$ resistant mutants that arose in the $\log$ phase could form colonies. The distribution of resistant colonies was highly variable and compatible with the hypothesis that mutant bacteria formed in the growing population before it encounters the selective agent. From the paper of Luria and Delbrück [2] a dogma of Neo-Darwinism has been that mutations are random events which form only in dividing bacteria. In 1988, Cairns and coworkers [3] challenged this dogma; they confirmed previous studies by Ryan and Shapiro $[4,5]$ showing that mutations can arise in apparently static bacterial populations when subjected to non-lethal selective pressure and suggested that only selected mutations, not deleterious or neutral ones, appeared in the population under selective pressure. In their experimental system Lac- cells plated on a lactose-containing medium survive and eventually perform mutations which are immediately expressed giving a selective advantage. This approach was very different from the one previously used by Luria and Delbrück [2] where cells sensitive to the phage immediately died when plated on the selective medium.

After that work many papers have been published about mutagenesis in microorganisms under non-lethal selective pressure. This review will deal with the results obtained in $S$. cerevisiae. To avoid any possible confusion, throughout this paper we name "adaptive mutagenesis" the process by which advantageous mutations are produced during selection even though other, non-selected, mutations are produced as well. We name "directed mutagenesis" the process which produces mutations in genes that give an adaptive phenotype that is mutations directed toward a useful goal. The experimental systems will be described in some detail to allow those who approach this field a better understanding of the results. Then we will move to the "State of the Art" with particular attention to genetic mechanisms of adaptive/directed mutagenesis. Finally, in the "perspective" section we will address the relevance of the phenomenon for the evolution of microbial populations in nature and for the onset of tumor processes in nonproliferating cells or non-growing tissues. 


\section{THE EXPERIMENTAL SYSTEM}

\section{Tests for adaptive mutation}

To prove the existence of adaptive mutations a non-lethal selective environment is required which allows cells to experience mutational events and to manifest them. In this respect the Luria and Delbrück fluctuation test [2] was not adequate. The selective agent in this test was bacteriophage T1 which destroyed cells in a short time. A more suitable approach is the one used by Ryan [4] who monitored the appearance of His $+E$. coli cells in His- cultures which did not multiply in a medium devoid of histidine. Cells ceased to increase in number after about 24 hours when they had barely doubled. Nonetheless, new His + colonies continued to appear with time. Since then, the reversion from auxotrophy to prototrophy has been used as a system to study adaptive mutation in bacteria as well in the yeast $S$. cerevisiae. In E.coli, however, the most thoroughly used reversion system makes use of the FC40 strain; this strain cannot utilize lactose but it reverts to Lac+ when lactose is the only carbon and energy source [6].

A demonstration of adaptive mutagenesis requires some experimental proofs:

i) To be considered adaptive, mutations must form after cells encountered the selective growth-limiting environment in which the mutations allow growth. Steele and Jinks-Robertson [7] studied the reversion to prototrophy of a $S$. cerevisiae strain containing the lys $2 \mathrm{del}$ taBgl allele (auxotroph for lysine, Lys-). To determine whether reversions occur before or after selective plating on minimal medium without lysine, they analyzed the data of a fluctuation test. Table 1 shows the results obtained in one of their experiments with 22 cultures

Таблища 1

Accumulation of Lys + revertants in a Lys- strain plated on minimal medium. The data from 22 independent cultures are shown (modified from $[7]$ ).

\begin{tabular}{|c|c|c|c|c|c|c|}
\hline \multirow{2}{*}{$\begin{array}{l}\text { Cells plated } \\
\left(\times 10^{7}\right)^{*}\end{array}$} & \multicolumn{6}{|c|}{ Number of newly arising colonies on day: } \\
\hline & 3 & 4 & 5 & 6 & 7 & 8 \\
\hline 105 & 24 & 9 & 6 & 5 & 6 & 0 \\
\hline 84 & 8 & 16 & 10 & 7 & 5 & 2 \\
\hline 93 & 7 & 10 & 5 & 2 & 4 & 7 \\
\hline 80 & 7 & 11 & 5 & 5 & 4 & 4 \\
\hline 79 & 12 & 18 & 6 & 3 & 5 & 6 \\
\hline 108 & 13 & 9 & 6 & 1 & 4 & 7 \\
\hline 80 & 7 & 23 & 6 & 5 & 3 & 2 \\
\hline 81 & 2 & 5 & 2 & 3 & 4 & 1 \\
\hline 90 & 10 & 16 & 8 & 3 & 4 & 2 \\
\hline 82 & 27 & 14 & 5 & 2 & 3 & 4 \\
\hline 80 & 81 & 14 & 2 & 4 & 13 & 6 \\
\hline 95 & 105 & 8 & 6 & 5 & 4 & 4 \\
\hline 99 & 10 & 11 & 2 & 3 & 4 & 4 \\
\hline 78 & 38 & 13 & 3 & 2 & 4 & 2 \\
\hline 86 & 91 & 14 & 7 & 7 & 5 & 3 \\
\hline 86 & 6 & 17 & 6 & 7 & 7 & 2 \\
\hline 78 & 37 & 23 & 3 & 6 & 3 & 2 \\
\hline 79 & 17 & 21 & 10 & 4 & 3 & 3 \\
\hline 94 & 34 & 23 & 7 & 8 & 4 & 0 \\
\hline 95 & 8 & 5 & 1 & 3 & 2 & 5 \\
\hline 83 & 8 & 8 & 1 & 3 & 2 & 5 \\
\hline 93 & 10 & 13 & 3 & 4 & 4 & 1 \\
\hline
\end{tabular}

* Each number given in the table represents the sum of the Lys + colonies obtained from each independent culture for a given day after plating on the selective medium. Each culture was split in 10 dishes with $10^{8}$ cells per dish. 


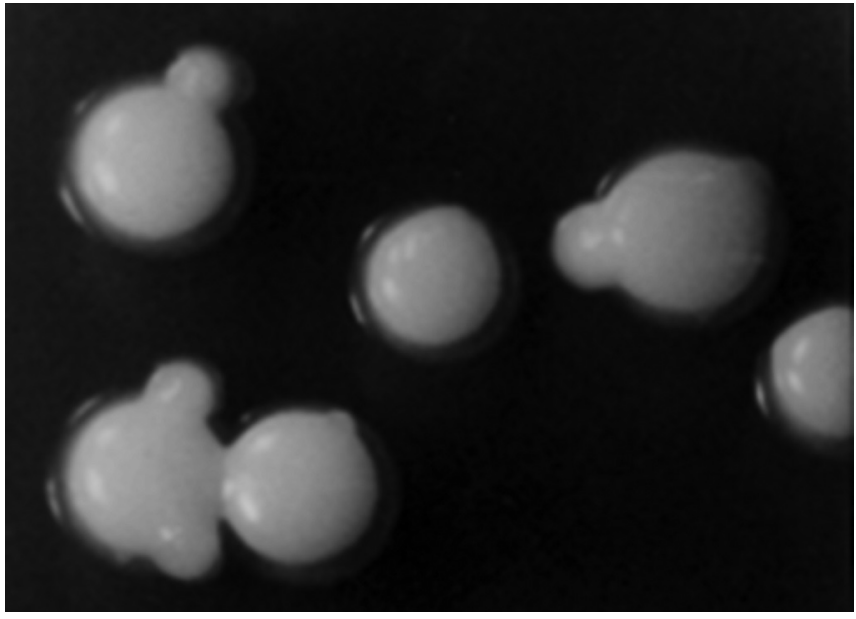

Figure 1. His + papillae on His- colonies grown on minimal medium with a limiting amount of histidine. In a Hall's test [9] yeast auxotroph cells are plated on minimal medium with a limited amount of the required nutrient and dishes are incubated. Colonies develop and stop growing a few days after plating; revertants form well distinguishable papillae.

[7]. Each entire overnight culture was plated in $0,1 \mathrm{ml}$ aliquots on 10 selective plates with approximately $10^{8}$ cells/plate. Each number given in Table 1 represents the sum of Lys + colonies on all 10 plates for a given day after plating on the selective medium. The first prototrophic colonies begun to appear on day 3 after plating ("early appearing" revertants) and the number of revertants increased in the following days. The numbers of revertants that newly appeared after day 3 ("late appearing" revertants) were similar in all cultures irrespective of the fact that some cultures showed many more "early appearing" revertants than others. By means of a reconstruction test the authors verified that the late appearance of Lys + colonies was not due to their low reduced growth with respect to "early appearing" revertants. Approximately $10^{\circ}$ cells of Lys + revertants isolated at definite times during the experiments were plated on the selective medium in the presence of $10^{8}$ cells of a strain containing non-revertable lys $21:: U R A 3$ allele. Most revertants formed colonies within three days after plating; therefore "late revertants" were not slowly growing logarithmic phase mutants. To verify whether mutations occurred during the non-selective logarithmic phase and/ or after selective plating, Steele and Jinks-Robertson [7] examined the distribution of revertants among independent cultures by statistical analysis. They concluded that "late appearing" revertants formed after selective plating. Hall $[8,9]$ devised a different experimental system which can be used in bacteria as well as in yeast. In his paper "Selection-induced mutations occur in yeast" [9] he studied the reversion from auxotrophy for histidine to prototrophy in a strain with a His- allele where the methionine encoding codon AUG has been mutated to isoleucine encoding codon AUU. It can revert either by direct reversion of AUU to AUG or by suppression. To measure the mutation rate under histidine starvation approximately 125 cells were plated on minimal medium containing a growth limiting histidine concentration and the plates were incubated for several days. On day 3 of incubation the maximum number of viable cells per colony was reached $\left(3,5 \times 10^{5}\right.$ cells per colony $)$. His + revertant papillae began to appear on day 6 and continued to appear for several days. Papillae can be easily scored at the microscope as shown in Figure 1, kindly provided by G. Morpurgo. An important point is to determine when the mutational events arose. If they arose during the logarithmic phase, a prototroph normal-size colony develops on plates. Instead, papillae can result either from revertant cells, which arose during the growth of the colonies, or from revertants, which arose after cells stopped dividing. It's possible to discriminate between these two possibilities by reconstruction experiments as described by Hall [9]. He plated on minimal medium drops of dense suspensions containing about $10^{6}$ cells of a His-, non-revertable strain plus approximately one His + revertant cell; true revertants formed papillae within 48 hours. In contrast, suppressor mutants required three to four days to form papillae on mutant colonies. ii) Evaluation of cell multiplications and survival. Auxotroph cells can multiply on selective medium in the first days after plating. This would occur for several reasons. Traces of the nutrient required for auxotroph cells can be present in the medium; therefore, in our experience, the use of purified agar is recommended. Notwithstanding this, some multiplications may still occur. Achilli and co-workers [10] showed that two adenine auxotroph strains (Ade-) behaved very differently on adenine-free medium. One of them (de3-01) is defective in the 3'5 ' exonuclease activity of polymerase delta, the other is wild-type. The strain de 3-01 stopped dividing on day 3 after plating; the wild type strain continued to multiply slowly and its survival was higher than that of the exonuclease deficient strain.

In general, yeast strains, especially mutators, are changeable with time in culture and different populations can substitute the original one (storing them at $-80{ }^{\circ} \mathrm{C}$ and sub-cloning by single cell may help to overcome this problem). Cells may even resume growth many days after plating on the selective medium: nutrients can be excreted by prototroph revertants which should be removed from plates as soon as they are formed, and by dead cells, a phenomenon known as "cannibalism" (Figure 2).

\section{ESTIMATION OF MUTATION RATES}

Growth-dependent mutation rates are given as the number of mutants per cell per generation (or division). For adaptive mutations, rates should be given as mutants 

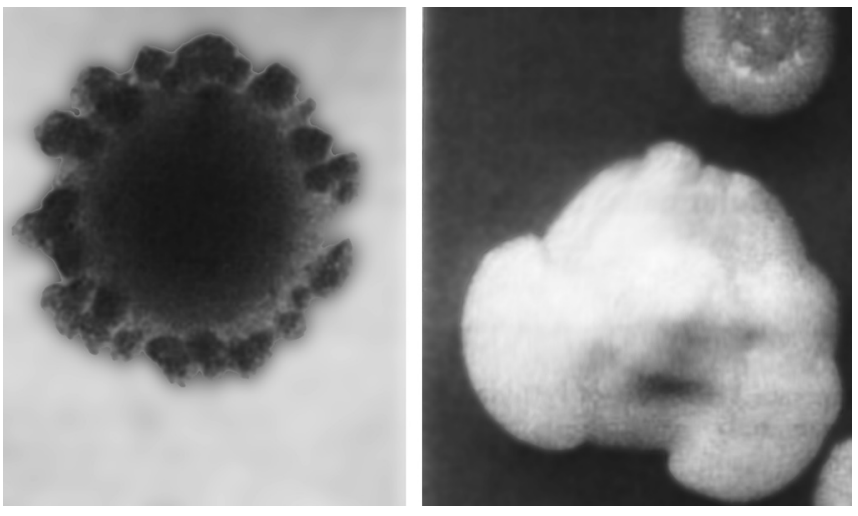

Figure 2. The appearance of His- colonies on minimal medium with a limited amount of histidine on day 12 after plating. A colony showing small His- papillae due to "cannibalism" (left); a colony showing overgrowth induced by the excretion of histidine by not-removed His+ papillae. (Modified from [11]).

per viable cell per physical time (hour or day). Therefore, the time a mutant requires to form a visible colony (or papilla) and the fraction of viable cells should be determined experimentally. It is not always possible to determine these parameters with accuracy; when it is, the adaptive mutation rate on day $\mathrm{n}$ is given by the number of revertants which appeared on that day per viable cells on day $n-x$ where $x$ is the time a revertant takes to form a visible colony (or papilla). Adaptive mutant growth rates may be different. Then the estimation of mutation rates cannot always be done as mentioned previously. Achilli with coworkers [10] showed that the growth rate of Ade+ revertants selected on adenine free medium from an Ade- strain were lower than that of the parental strain and different from each other. That made the exact estimation of adaptive mutation rates on day $\mathrm{n}$ almost impossible; therefore the authors gave the reversion rate as ratio of the Ade+ revertants to cells plated.

\section{Tests for directed mutation}

In their original paper, Cairns and coworkers [3] provided evidence that the rate of beneficial mutations was higher than the rate of mutation which was adaptively neutral under conditions of the test and hypothesized that mutations could be directed toward a useful goal. To test the hypothesis of directed mutations, they estimated the rate of mutation of a neutral marker, valine-resistance. Neutral markers have been used in yeast as well. For example, Marini and coworkers [11] studied the reversion from auxotrophy to prototrophy for histidine with the reversion from auxotrophy for tryptophan (Trp-) to prototrophy (Trp+) as neutral marker. Also, Achilli et al. [10] tested for the presence of temperature-sensitive and nutritional mutants in a study where auxotrophic Ade- cells were starved for adenine.
The choice of a neutral marker should take into consideration any available information about its molecular nature and some possible technical artifacts. We would like to give just two examples: if the marker under selection is a frameshift allele, base-substitution alleles should not be used as neutral markers (and vice-versa). Nutrients added to the medium also have to be considered carefully: MacPhee [12] criticized Cairns and coworkers [3] who used valine-resistance as a neutral marker. Those experiments to test the frequency of valine-resistant mutants were made using a different carbon source (glucose) with respect to the one used in experiments for Lac- to Lac+ reversion (lactose). Lastly, the positions of the marker under selection and of the neutral ones are relevant. Both should be on the main chromosomes or on accessory DNA molecules such as plasmids. In fact, different mechanisms may act on different DNA molecules.

\section{THE STATE OF THE ART}

\section{Do adaptive mutations exist in yeast? Are they directed?}

Modern data strongly suggest that adaptive mutations in yeast are induced as a response to nutritional stress. Some molecular mechanisms have been partially elucidated, although not in such detail as it was done in E.coli FC40 [13, 14, 15, 16]. A more controversial point is the directed mutations hypothesis. Hall [9] concluded that the reversion from His- auxotrophy to His + prototrophy could be specific for the selective pressure, although Hall considered that more experimental proofs are necessary to prove directed mutagenesis in yeast. On the contrary, Marini with coworkers [11] and Achilli with coworkers [10] showed that adaptive mutagenesis is not directed in yeast. It is worth mention that in E.coli the hypothesis of directed mutagenesis was fairly eliminated by negative evidences obtained by Foster [17] and Foster and Cairns [18] except for mutations directed by transcription [14, 15, 19], which will be discussed below.

\section{Mechanisms of adaptive mutagenesis}

Mutations are generating either during DNA replication or during DNA repair. In adaptive mutagenesis DNA repair should be the most relevant since cells do not replicate or replicate poorly when nutrients become limiting. Indeed, the integrity of the replicating genome is constantly endangered by a variety of spontaneous lesions [20,21], which could occur in starving cells as 

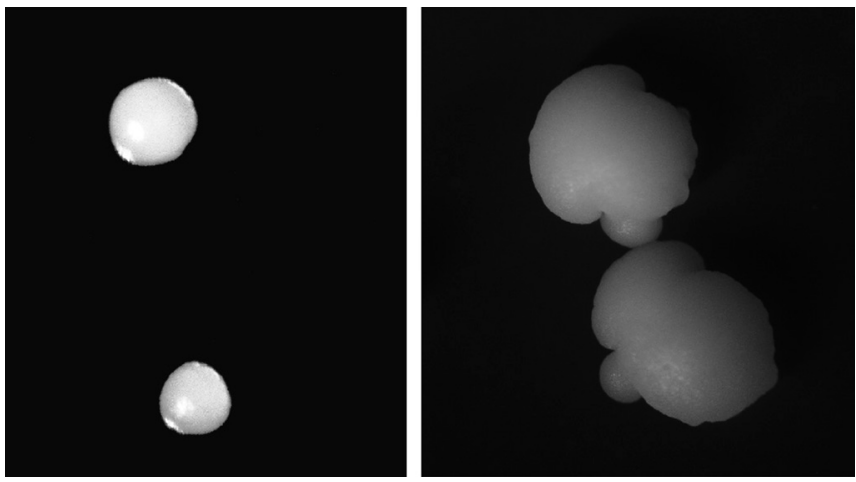

Figure 3. Effect of adenine starvation on partially reverted Ade + colonies.

A colony of the strain de 3-01 (see text) only partially reverted to adenine prototrophy was grown for six days on adenine-free medium (left); four days later, the same colony (right) showed papillae outgrowing from the colony surface (unpublished results).

well [22, 23]. Both error-free and error-prone DNA repair pathways could be involved in the repair of lesions during starvation for nutrients. We will briefly summarize the most relevant papers about pathways either counteracting or promoting adaptive mutagenesis.

\section{Which DNA repair pathways counteract adaptive mu- tagenesis?}

First, we will briefly review pathways preventing adaptive mutations. The role of nucleotide excision repair (NER) (see [24, 25] for review) in adaptive frameshift mutations has been studied by Heidenreich with coworkers [26]. They used yeast strains, where the genes RAD16 (involved in the global genome NER), RAD26 (involved in the transcription coupled NER) and RAD14 (an essential factor for both NER pathways) were disrupted. These defects in NER resulted in increased frequencies of adaptive mutations with the highest values in the rad14 strain. Therefore NER processes spontaneous lesions in starved, cycle-arrested cells as it does in proliferating cells. Storchová and coworkers [27] and Cejká and coworkers [28] analyzed the effect of mutations of the RAD6 repair pathway which is involved in the repair of bulky adducts to DNA and in mutagenesis. The pathway is controlled by many genes whose epistatic relationships showed that it can be dissected in at least three sub-pathways. The pivotal gene of these pathways is the RAD6 gene whose product controls many cellular processes in addition to DNA repair and mutagenesis (see for reviews [29-33]. Storchová with coworkers [27] in poliauxotrophic strains defective in the RAD6 pathway showed an enhanced accumulation of Ade + revertants during adenine starvation and of Trp+ revertants during tryptophan starvation. Cejká with coworkers [28] gave more insights on the role of the RAD6 pathway in adaptive mutagenesis. They studied the rever- sion of the amber allele ade2-101 in strains with different alleles of the RAD6 gene and in strains mutated at the loci REV3, RAD5, RAD 18 and MMS2, already known to be involved in the $R A D 6$-controlled pathway. Their results showed that the RAD6 pathways are active not only in replicating cells but also in starved cells and that they keep both logarithmic and adaptive mutation rates at a basal level (for the role of the REV3 gene and polymerase zeta see below).

The role of polymerases delta and epsilon in adaptive mutagenesis was studied by several authors [34, 35, 10]. Their data show that polymerases delta and epsilon are involved in the control of mutability in non-growing, starved cells; because adaptive mutation frequencies were enhanced in all mutant strains. Exceptionally high rates were observed in the strain de3-01, defective in the 3'-5' exonuclease activity of polymerase delta, starved for adenine. On day 16 after plating on minimal medium without adenine, the reversion rate from Ade- to Ade+ was $1 \%$, given as the rate of the total number of Ade+ revertants to number of plated cells (see above). Most Ade + revertants showed additional nutritional requirements and $51 \%$ were temperature sensitive. Among Ade- survivors $66 \%$ were nutritional mutants and $39 \%$ were temperature sensitive. Instead, the reversion rate from Trp- to Trp + was much lower. Therefore, it has been concluded that adenine starvation is highly mutagenic in yeast and it induces genome-wide hyper mutagenesis in the strain devoid of the polymerase delta proofreading activity [10]. The mutagenic effect of adenine starvation in de 3-01 cells is well documented in Figure 3. Most of Ade + revertants (on de3-01 background) grew poorly on minimal medium either because of additional mutations [10] or because of partially restored prototrophy. When they were plated on minimal medium without adenine colonies developed (Figure 3 left) and while the further incubation papillae formed on them (Figure 3 right). We interpreted these observations as follows: the partially reverted Ade + cells of the colonies were still sensitive to the mutagenic effect of adenine starvation; therefore some of them acquired new mutations which endowed them with higher growth ability (unpublished results).

In order to understand the role of yeast mismatchrepair (MMR) in adaptive mutagenesis, Halas and coworkers [36] studied the effects of deletions in the MSH2, MSH3 and MSH6 genes (see for review [37-39]). All of them resulted in elevation of the mutation rate, which was more evident in the strain where the $\mathrm{MSH} 2$ gene was disrupted. On the base of that data, it was concluded that MMR prevents the formation of adaptive mutations.

An increase of adaptive mutations frequency has also been observed following the inactivation of the RAS2 gene [40]. In yeast the RAS2 gene product is involved in the cAMP-protein kinase A signaling. This pathway is fundamental for control of metabolism, stress resistance 
and cellular proliferation in connection with the available nutrient conditions $[41,42]$. It could be interesting to investigate the role of the cAMP-protein kinase A signaling pathway in adaptive mutagenesis to understand how it is regulated by the environment.

\section{Which DNA repair pathways promote adaptive muta- genesis?}

To answer this question mutants defective in translesion synthesis (TLS) and in the repair of double strand breaks (DSB) have been used. Some lesions severely endangered the genome if left unrepaired before DNA replication starts because replicating DNA polymerases are not able to bypass non-coding lesions. TLS polymerases can insert either the correct or the wrong nucleotides opposite the non-coding lesion, so that error-free or error-prone bypass can result. In yeast DNA polymerase eta encoded by the RAD30 gene preferentially inserts two adenines opposite of the thymine dimers, therefore it is considered an error-free polymerase in respect to UV mutagenesis. However, pol eta has very low fidelity when copying undamaged DNA templates. It is important for UV-induced mutagenesis in several yeast assays [43-46]. Polymerase zeta is thought to be responsible for more than half of the spontaneous mutations in replicating cells. It is also responsible for the increased mutation rates during transcription. It consists of two subunits, one of them is encoded by the $R E V 3$ gene, the other one by the $R E V 7$ gene. $R E V 3 \mathrm{p}$ is the catalytic subunit; the role of REV7p is not clear yet. Polymerase zeta functions with REV1p in the by-pass of abasic sites; REV1p has a deoxicytidyl activity which inserts $C$ in front of abasic sites with polymerase zeta doing the extension (see for review [47, 48]). Heidenreich with co-workers investigated adaptive reversions in lysine auxotroph strains with the frameshift lys 2 deltaBgl allele. In cells starved for lysine, they showed that when NER-processed lesions were increased by NER deficiency or by UV irradiation a mutagenic polymerase zeta-dependent pathway was activated [26]. On the basis of these results and because of the epistatic relationship between REV3 and REV1, Heidenreich with coworkers [46] propose that in cell cycle arrested cells, when NER does not operate or when it is saturated by excessive damage, TLS by polymerase zeta and Revlp can generate adaptive frameshift mutations. Non-homologous end joining (NHEJ) ( see for review [49]) accounts for $50 \%$ of adaptive frameshift mutations, but not for replicationdependent mutations, as shown by the decrease in adaptive mutation rates in strains where the DNL4 or YKU70 genes have been deleted [50]. The DNL4 gene encodes the catalytic subunit of DNA ligase IV, which is involved in the sealing of DSB. The YKU70 gene encodes a subunit of the telomeric Ku complex (Yku70p-Yku80p), involved in telomere length maintenance, structure and telomere position effect; it relocates to sites of double-strand cleavage to promote NHEJ during DBS repair (see for review [51, 52]). Gamma-rays induced adaptive frameshift reversions are also dependent on NHEJ as shown by Heidenreich and Eisler [53]. The NHEJ effect on adaptive frameshift mutagenesis suggests that in amino acids starved, cell cycle arrested cells, DBS repair is induced and is responsible for the generation of adaptive mutations. DBS repair can be processed by homologous recombination (HR), single strand annealing (SSA) and NHEJ. However, the data of Heidenreich and coworkers [50] clearly show that only NHEJ contributes to adaptive frameshift mutagenesis; indeed experiments with RAD54 and RAD52 deficient strains showed that HR and SSA do not promote adaptive mutations, instead they counteract it. The RAD54 gene product is a DNA-dependent ATPase which stimulates strand exchange by modifying the topology of double-stranded DNA. The RAD52 protein stimulates strand exchange and anneals complementary single-stranded DNA. Both gene products are involved in the repair of DSB in DNA during vegetative growth and meiosis [49]. The pathways promoting base-substitutions adaptive mutagenesis are less understood. Cejká with coworkers [28] showed that a deficiency for the REV3 gene (DNA polymerase zeta) did not significantly change the frequency of reversion of an ochre nonsense allele in a repair proficient strain, a result in line with those of Heidenreich et al. [26]. Instead the mutator phenotype of rad5 and rad 18 mutants is completely dependent on the REV3 gene product. The product of the RAD5 gene is a single-stranded DNA-dependent ATPase, involved in post-replication error-free repair [54]. The $R A D 18$ gene is necessary for the RAD6 gene repair functions: their products form a complex which targets damaged DNA and allow the repair. Therefore, Cejká with coworkers [28] suggested that the REV3 can act either in the sub-pathway mediated by the $R A D 18$ or in a subpathway mediated by the RAD5. The similar mutations rates observed in the rad6 single mutant and in the rad6 rev3 double mutant suggest that polymerase zeta is not responsible for mutagenesis in the rad6 background.

In conclusion, the available data show that: i) adaptive frameshift mutagenesis could be kept at the wild type level by NER and MMR [26, 36] with the involvement of DNA polymerases delta and epsilon [34, 35]. Also, it could be enhanced when these pathways are saturated and lesions of DNA in non-dividing cells are processed through error-prone pathways such as TLS or NHEJ [26, 50 ]; ii) adaptive base-substitution mutagenesis is kept at wild type levels by the RAD6 error-free pathway [27, 28 ] with an important role played by the polymerase delta proofreading activity [10]. Polymerase zeta could be responsible for the low increase in adaptive base-substitution mutagenesis in a wild type background, although other genes could be involved as well [28]. 
Is the mechanistic origin of adaptive mutations different from that of replicating cells?

Most data suggest that at least a portion of adaptive mutations are formed by different mechanisms than those operating in replicating cells. As discussed previously [50], the products of the DNL4 and YKU70 genes in yeast are responsible for half of the adaptive frameshift mutations at the lys2deltaBgl allele but not for replication-dependent mutations. Heidenreich and Wintersberger [55] compared sequences from late appearing, adaptive Lys + revertants with sequences of revertants that arose in proliferating cells. Different mutational spectra were observed: in revertants from proliferating cells nucleotide gain and loss were balanced; instead, in adaptive revertants, simple deletions in mononucleotide repeats were the prevalent event. Then slippage of polymerases, possibly polymerase delta, is likely to occur during DNA repair synthesis in cell cycle arrested, lysine-starved cells [55].

\section{Transcription and adaptive directed mutagenesis}

It has been shown that specific starvation conditions target genes for derepression and increased rates of transcription and mutation in bacteria [56-58]. A positive correlation between transcription and mutability has also been observed in yeast [59]. Transcription associated mutagenesis in yeast requires the activity of the error prone polymerase zeta while it is counteracted by NER or recombinational pathways [59, $60]$. A distinctive mutation spectrum has been found to be associated with high transcription in yeast suggesting that high transcription may alter the mechanisms of mutagenesis [61]. Then it could be that in nature, where cells divide poorly because of adverse conditions, beneficial mutations can arise as a response to stresses that target specific genes for transcription. Another way in which mutations could be at least loosely targeted to specific genes is translesion synthesis by RNA polymerases, that is bypass of lesions by RNA polymerases with a misincorportation event [62]. Translesion synthesis has been demonstrated in vitro and in vivo in non-dividing E. coli cells; Viswanathan and coworkers [63] showed that bypass of uracil on the template strand of the luciferase gene allows the synthesis of the luciferase protein. Doetsch [62] proposed a "Retromutagenesis model" which can be relevant to adaptive mutagenesis in starved cells. Transcriptional bypass of a DNA lesion could lead to mutated proteins which allow cells to switch from a nongrowth to a growth state. Then DNA replication could start and, if lesions are not repaired, the mutation could be fixed.

\section{PERSPECTIVE}

In nature, micro-organisms often experience adverse conditions and divide poorly. Most mutations are detrimental but, in a stressful environment, maintaining or even increasing variation could allow some cells to acquire a phenotype most likely to evolve in that environment. In this context, adaptive mutations are particularly relevant since advantageous mutations are produced transiently during selection, though other, non-selected, mutations may occur at the same time. A transient increase in mutation rate would be particularly advantageous with respect to the acquisition of a stable mutator phenotype which can burden survivors for a long time. Cells with a reduced fitness may form during adaptive mutagenesis [10] but since their mutability is normal, the genetic burden could be minimized in the next generations. The genetic burden could be irrelevant in natural yeast strains which are diploid and can sustain more deleterious mutations as it has been shown in Aspergillus nidulans by Pimpinelli with coauthors [64]. The authors repeatedly treated conidia with the base-analog 6-hydroxlaminopurine and showed that after 12 cycles of treatment heterozygous conidia differed from each other for at least 10 recessive lethal mutations and therefore there are probably hundreds of mutations without any apparent reduction in vitality.

In conclusion, according to many authors (see for review $[14,65]$ ), stress-induced adaptive mutagenesis could be an advantageous process which specifically evolved because it allows microbial populations to survive in an adverse environment. A different view, based on the amplification model proposed for the Lac- to Lac+ reversion in E. coli $[66,67]$, has been recently reviewed by Roth and coworkers [16]. According to the authors, mutations under stressful conditions do not arise by way of ad hoc mechanisms but are the consequences of common mutagenesis pathways acting on growing subpopulations of cells. Further efforts should be made to unravel the mechanisms of mutagenesis in yeast cells under stressful environmental conditions; they might help to understand evolution in unicellular eukaryotes and the initiation of carcinogenesis. Many genes shown to be involved in adaptive mutagenesis in yeast have counterparts in humans where they are implicated in pathogenesis and it is known that cells in homeostatic, non-proliferating tissues might mutate and begin to multiply, initiating tumorigenesis.

\section{ACKNOWLEDGEMENTS}

We thank Dr. Youri I. Pavlov for reading the manuscript and Prof. Giorgio Morpurgo for helpful discussion and for providing Figure 1. We are grateful to the University of Perugia (Italy), the University of Nebraska Medical Centre (U.S.A.) and the University of Dubna (Russia) for the Cultural Agreement which contributes to the exchange for researchers and, therefore, ideas between countries. 


\section{REFERENCES}

1. Hayes $W$. The Genetics of Bacteria and their Viruses. Blackwell Scientific Publications, Oxford. - 1964. Vol. 177. - 198 p.

2. Luria S. E. and Delbrück M. Mutations of bacteria from virus sensitivity to virus resistance // Genetics. - 1943. Vol. 28. - P. 491-511.

3. Cairns J., Overbaugh J. and Miller S. The origin of mutants // Nature. - 1988. - Vol. 335. - P. 142-145.

4. Ryan F. J. Spontaneous mutations in non-dividing bacteria // Genetics. - 1955. - Vol. 40. - P. 726-738.

5. Shapiro J. A. Observations on the formation of clones containing araB-lacZ fusions // Mol. Gen. Genet. — 1984. Vol. 194. - P. 79-90.

6. Cairns J. and Foster P. L. Adaptive reversion of a frameshift mutationinEscherichiacoli//Genetics. - 1991. - Vol.28. P. 695-701.

7. Steele D. F. and Jinks-Robertson S. An examination of adaptive reversion in Saccharomyces cerevisiae // Genetics. 1992. - Vol. 132. - P. 9-21.

8. Hall B. G. Spontaneous point mutations that occur more often when advantageous than when neutral// Genetics. - 1990. Vol. 126. - P. 5- 16.

9. Hall B. G. Selection induced mutations occur in yeast // Proc. Natl. Acad. Sci. USA. - 1992. - Vol. 89. - P. 4300-4303.

10. Achilli A., Pavlov Y. I., Matmati N. et al., The exceptionally high rate of spontaneous mutations in the polymerase delta proof-reading exonuclease deficient S.cerevisiae strains starved for adenina // BMC Genetics. - 2004. - Vol. 5. P. $34-44$.

11. Marini A., Morpurgo G. and Matmati N. Starvation in yeast increases non-adaptive mutation // Curr. Genet. - 1999. Vol. 35. - P. 77-81.

12. McPhee $D$. Is there evidence for directed mutation in bacteria? // Mutagenesis. - 1993. - Vol. 8. - P. 3-5.

13. Foster P. L. Adaptive mutation in Escherichia coli // J. Bacteriol. - 2004. - Vol. 186. - P. 4846-4852.

14. FosterP. L. Stress responses and genetic variation in bacteria // Mut. Res. - 2005. - Vol. 569. - P. 3-11.

15. Rosenberg S. M. Evolving responsively: adaptive mutation // Nat Rev Gene. - 2001. - Vol. 2. - P. 504-515.

16. Roth J. R., Kugeleberg E., Reams A. B. et al. Origins of Mutations Under Selection: The Adaptive Mutation Controversy // Annu. Rev. Microbiol. - 2006. - Vol. 60. P. 477-501.

17. Foster P. L. Nonadaptive mutations occur on the F' episome during adaptive mutations conditions in Escherichia coli // J. Bacteriol. — 1997. — Vol. 174. - P. 1711-1716.

18. Foster P. L. and Cairns J. Adaptive mutation of a lacZ amber allele // Genetics. - 1998. - Vol. 150. — P. 1329-1330.

19. Wright B. E. Stress-directed adaptive mutations and evolution // Mol. Microbiol. - 2004. - Vol. 52. - P. 643-650.

20. Maki H. Origins of spontaneous mutations: specificity and directionality of base-substitutions, frameshift and sequence- substitution mutagenesis // Annu. Rev. Genet. — 2002. Vol. 36. - P. 279-303.

21. Barnes D. E. and Lindahl T. Repair and genetic consequences of endogenous DNA base damage in mammalian cells // Annu. Rev. Genet. - 2004. - Vol. 38. - P. 445-476.

22. Bridges B. A. Mutation in resting cells: the role of endogenous DNA damage // Cancer Surv. - 1996. - Vol. 2. - P. 155-167.

23. Heidenreich $E$. and Wintersberger $U$. Starvation for specific aminoacid induces high frequencies of rho- mutants in Saccharomyces cerevisiae // Curr. Genet. - 1997. Vol. 31. - P. 408-413.

24. Prakash S. and Prakash. L. Nucleotide excision repair in yeast // Mut. Res. - 2000. - Vol. 451. - P. 13-24.

25. Abdulovic A., Kim N. and Jinks-Robertson S. Mutagenesis and the three R's in yeast // DNA repair. - 2006. - Vol. 5. P. 409-421.

26. Heidenreich E., Holzmann V. and Eisler H. Polymerase zeta dependency of increased adaptive mutation frequencies in nucleotide excision repair-deficient yeast strains // DNA Repair. - 2004. - Vol. 3. - P. 395-402.

27. StorchováZ., Rohas A.P., JanderováB., et al. The involvement of the RAD6 gene in starvation-induced reverse mutations in Saccharomyces cerevisiae // Mol. Gen. Genet. — 1998. Vol. 258. - P. 546-552.

28. Ceiká P., Vondrejs V., Storchová S. Dissection of the functions of the Saccharomyces cerevisiae RAD6 postreplicative repair group in mutagenesis and UV sensitivity // Genetics 2001. - Vol. 159. - P. 953-963.

29. Prakash $L$. The structure and function of RAD6 and RAD18 DNA repair genes of Saccharomyces cerevisiae // Genome. 1989. - Vol. 31. - P. 597-600.

30. Lawrence C. The RAD6 DNA repair pathway in Saccharomyces cerevisiae: what doesit do, and how doesit do it? //Bioessays. 1994. - Vol. 16. - P. 253-258.

31. Watts F. Z. Sumoylation of PCNA: Wrestling with recombination at stalled replication forks // DNA Repair. - 2006. Vol. 5. - P. 399-403.

32. Ulrich H. D. Mutual interactions between the SUMO and ubiquitin systems: a plea of no contest // Trends Cell Biol. 2005. - Vol. 15. - P. 525-532.

33. Ulich H. D. The RAD6 pathway: control of DNA damage bypass and mutagenesis by ubiquitin and SUMO // Chembiochem. - 2005. - Vol. 6. - P. 1735-1743.

34. Baranowska H., Policinska Z. and Jachymezyk W. Y. Effects of the CDC2 gene on adaptive mutation in the yeast Saccharomyces cerevisiae // Curr. Genet. - 1995. Vol. 28. - P. 521-525.

35. Babudri N., Pavlov Y. I., Matmati N., et al. Stationary- phase mutations in proofreading exonuclease-deficient strains in the yeast Saccharomyces cerevisiae // Mol. Gen. Genomics. 2001. - Vol. 265. - P. 362-366.

36. Halas A., Baranowska H., Policinska Z. The influence of the mismatch-repair system on stationary-phase mutagenesis in the yeast Saccharomyces cerevisiae // Curr. Genet. 2002. - Vol. 42. - P. 140-146. 
37. Modrich $P$. and Lahue $R$. Mismatch repair in replication fidelity, genetic recombination, and cancer biology // Ann. Rev. Biochem. - 1996. - Vol. 65. - P. 101-133.

38. Harfe B. D. and Jinks-Robertson S. DNA mismatch repair and genetic instability // Ann. Rev. Genet. - 2000. Vol. 34. - P. 359-399.

39. Kunkel T. A. and Erie D. A. DNA mismatch repair // Ann. Rev. Biochem. - 2005. - Vol. 74. - P. 681-710.

40. Storchov Z. and Vondrejs $V$. Starvation-associated muta-genesis in yeast Saccharomyces cerevisiae is affected by Ras2/cAMP signaling pathway // Mut. Res. - 1999. - Vol. 16. - P. 59-67.

41. Thevelein J. M. and de Winde J. H. Novel sensing mechanisms and targets for the cAMP - protein kinase A pathway in the yeast Saccharomyces cerevisiae // Mol. Microbiol. 1999. - Vol. 33. - P. 904-918.

42. Estruch F. Stress-controlled transcription factors, stressinduced genes and stress tolerance in budding yeast // FEMS Microbiol. Rev. - 2000. - Vol. 24. - P. 469-486.

43. Bresson A. and Fuchs R. P. Lesion bypass in yeast cells: Pol eta participates in a multi-DNA polymerases process // EMBO J. - 2002. - Vol. 21. - P. 3881-3887.

44. Zhang H. and Siede W. UV-induced TC transition at a TT photoproduct site is dependent on Saccharomyces cerevisiae polymerase eta in vivo // Nucleic Acid Res. - 2002. Vol. 30. - P. 1262-1267.

45. Kozmin S. G., Pavlov Y. I., Kunkel T. A., et al. Roles of Saccharomyces cerevisiae DNA polymerases Poleta and polzeta in response to irradiation by simulated sunlight // Nucleic Acid Res. - 2003. - Vol. 31. - P. 4541-4552.

46. Heidenreich E., Eiser $H$. and Steinboeck F. Epistatic participation of REV1 and REV3 in the formation of UVinduced frameshift mutations in cell cycle arrested cells // Mut. Res. - 2006. - Vol. 593. - P. 187-195.

47. Abdulovic A. L. and Jinks-Robertson S. The in vivo characterization of translesion synthesis across UV-induced lesions in Saccharomyces cerevisiae: insights into Pol zeta-and Pol eta- dependent frameshift mutagenesis // Genetics. 2006. - Vol. 172. - P. 1487-1498.

48. Lawrence C. W. and Maher V. M. Eukaryotic mutagenesis and translesion replication dependent on DNA polymerases zeta and Rev1 protein // Biochem. Soc. Trans. - 2001. Vol. 29. - P. 187-191

49. Daley J. M., Palmbos P. L., Wu D. et al., Nonhomologous end joining in yeast // Annu. Rev. Genet. - 2005. — Vol. 39. P. $431-451$.

50. HeidenreichE., Novotny R., KneidingerB., et al. Non-homologous end joining as an important mutagenic process in cell cycle-arrested cells. // EMBO J. - 2003. - Vol. 22. - 2274-2283.

51. Krog B. O. and Symington L. S. Recombination proteins in yeast // Annu Rev. Genet. - 2004. - Vol. 38. P. $233-271$.

52. Aylon Y. and Kupiec M. DSB repair: the yeast paradigm // DNA Repair. - 2004. - Vol. 3. - P. 797-815.

53. Heidenreich E. and Eisler H. Non-homologous end joining dependency of gamma-irradiation induced adaptive frameshift mutation formation in cell cycle-arrested yeast cells. // Mut. Res. - 2004. - Vol. 556. - P. 201-208.

54. Xiao W., Chow B. L., Broomfield S., et al. The Saccharomyces cerevisiae RAD6 group is composed of an error-prone and two error-free postreplication repair pathways // Genetics. 2000. - Vol. 155. - P. 1633-1641.

55. Heidenreich $E$. and Wintersberger $U$. Adaptive reversions of a frameshift mutation in arrested Saccharomyces cerevisiae cells by simple deletions in mononucleotide repeats // Mut. Res. - 2001. - Vol. 473. - P. 101-107.

56. Wright B. E., Longacre A. and Reimers J. M. Hypermutation in derepressed operons of Escherichia coli K12 // Proc. Natl. Acad. Sci. USA. - 1999. - Vol. 96. - P. 5089-5094.

57. Wright B. E. A biochemical mechanism for non-random mutations and evolution // J. Bacteriol. - 2000. Vol. 182. - P. 2993-3001.

58. Reimers J. M., Schmidt K. H., Longacre A. Increased transcription rates correlate with increased reversion rates in leuB and argH Escherichia coli auxotrophs // Microbiology. 2004. - Vol. 150. - P. 1457-1466.

59. Datta A. and Jinks-Robertson S. Association of increased spontaneous mutation rates with high levels of transcription in yeast // Science. - 1995. - Vol. 26. - P. 16161619 .

60. Morey N. J., Greene C. N. and Jinks-Robertson S. Genetic analysis of transcription-associated mutations in Saccharomyces cerevisiae // Genetics. - 2000. Vol. 154. - P. 109-120.

61. Lippert M. J., Freedman J. A., Barber M. A. et al. Identification of a distinctive mutation spectrum associated with high levels of transcription in yeast // Mol. Cell. Biol. - 2004. Vol. 24. - P. 4801-4809.

62. Doetsch $P$. W. Translesion sythesis by RNA polymerases: occurrence and biological implications for transcriptional mutagenesis // Mut. Res. - 2002. - Vol. 510. - P. 131-140.

63. Viswanathan A., You H. J. and Doetsch P. W. Phenotypic change caused by transcriptional bypass of uracil in nondividing cells // Science. - 1999. - Vol. 284. - P. 159-162.

64. Pimpinelli S., Marini A., Babudri N. et al. 6-N-hydroxylaminopurine (HAP)-induced accumulation of variability in haploid and diploid strains of Aspergillus nidulans // Curr. Genet. - 1997. - Vol. 32. - P. 331-336.

65. Kivisaar $M$. Stationary phase mutagenesis: mechanisms that accelerate adaptation of microbial populations under environmental stress // Envirom. Microbiol. - 2003. Vol. 5. - P. 814-827.

66. Roth J. R., Andersson D. I. Adaptive mutations: how growth under selection stimulates $\mathrm{Lac}(+)$ reversion by increasing target copy number // J. Bacteriol. - 2004. - Vol. 186. P. $4855-4860$.

67. Roth J. R., Andersson D. I. Adaptive mutation: how growth under selection contributes to the origin of genetic diversity and explains the phenomenon of adaptive mutations // Res. Microbiol. - 2004. - Vol. 155. P. $342-351$. 